\title{
PACKeT Size OPTIMiZATION FOR ENERgY EFFICIENCY IN MULTIPATH FADING FOR WIRELESS BODY AREA NETWORK
}

\author{
Nattkorn Promwongsa and Teerapat Sanguakotchakorn \\ Department of Telecommunications, Asian Institute of Technology, Pathumthani, \\ Thailand
}

\begin{abstract}
Recently, Wireless Body Area Network (WBAN) has drawn vast interest to many researchers due to its potential in healthcare and medical fields. In this paper, we propose the energy efficiency model of Time Diversity communication in Rician and Rayleigh fading channels emphasizing the optimal packet size. The optimal packet size to maximize energy efficiency is investigated for on-body and in-body channels. Four modulation techniques are considered including the recently proposed 16-ary Quadrature Amplitude Position Modulation (QAPM). In addition, the closed-form expression of the energy efficiency model in multipath fading is derived. Here, three evaluation metrics including the energy efficiency, the normalized throughput and the average end-to-end delay are adopted and compared to the existing 1-hop and Cooperative communications as well as our previously proposed 2-hop communication. The results show that our proposed Time Diversity communication scheme outperforms the existing ones in deep fading channel except for the average end-to-end delay.
\end{abstract}

\section{KEYWORDS}

Packet Size Optimization, Energy Efficiency, 2-hop, Time Diversity, Multipath Fading, Wireless Body Area Network

\section{INTRODUCTION}

Wireless Body Area Network (WBAN) is one of the wireless networking technologies having a lot of applications in healthcare, medical field, sports, military and entertainments. It consists of wireless sensing nodes that are placed on, in or around the human body. Typically, WBAN nodes are classified into three groups: (i) Sensing nodes that comprise wearable and implanted nodes depending on their location on the human body. The former [1] are placed on the human body and mainly used to monitor a person's health conditions while the latter [2] are located inside the human body, and mainly used to collect image information inside the human body. (ii) Coordinator node $(\mathrm{CN})$ which is responsible for sending the control message to, and gathering information from the sensing nodes. (iii) Access point or monitoring station. In general, the communication types of WBANs can be classified into on-body and in-body channels. The on-body and in-body channels are the communications between wearable and coordinator nodes, and implanted and coordinator nodes, respectively. Due to the potential applications in WBANs, IEEEs have standardized IEEE 802.15.6 for WBANs [3] specifying physical and MAC layers.

Generally, energy efficiency is one of the challenging issues in WBANs, since WBAN nodes are expected to operate continuously for a long period of time even though they are small size, light weight and energy constraint. Moreover, replacing and recharging their batteries may not be practical when they are in operation. Similar to Wireless Sensor Networks (WSNs), 
multipath fading cannot be ignored in WBANs as well. In [4] and [5], multipath fading in WBANs is evaluated, and it is shown that multipath fading can be modelled closely by using Rician distribution in the on-body channel. In addition, in [6], Rician distribution is also used to model multipath fading in the on-body channel to evaluate the performance of a RAKE receiver.

It should be stated that WBAN has different channel characteristics from other networks. For example, in [2] a specific path loss model for WBANs is proposed because the signals are transmitted along or through the human body, which has the totally different path loss model from typical wireless channels. Furthermore, WBANs also have strict resource constraint and low transmission power [1]. Under these circumstances, packet size is one crucial factor that influences the energy efficiency in WBANs. By selecting the appropriate packet size [7]-[10] corresponding to network conditions, a packet error rate can be reduced, thus improving the energy efficiency. Another way to improve the energy efficiency in high path loss and shadowing fading channels is a multi-hop communication [11]-[14], because it provides more reliable and effective communication, comparing to a direct long-distance communication. Moreover, diversity technique is widely used as an efficient solution to mitigate the impact of multipath fading in WBANs [15]-[19].

Consequently, in [20] we proposed the energy efficiency model of ARQ protocol for 2-hop communication in order to select the energy-efficient packet size in WBANs. The average endto-end delay was also analyzed to evaluate the performance of our proposed 2-hop communication in [21]. Interestingly, the results show that our proposed 2-hop communication does not only significantly improve the energy efficiency, comparing to the existing 1-hop and Cooperative communications, but also provides the average end-to-end delay close to the Cooperative communication. However, the energy efficiency of Time Diversity communication and the normalized throughput performance have not been explored yet. Therefore, in this paper we extend our previous studies as follows: We first propose the energy efficiency model of Time Diversity communication in multipath fading. The optimal packet size to maximize the energy efficiency is then investigated. In addition to Binary Phase Shift Keying (BPSK) and 16ary Quadrature Amplitude Position Modulation (QAPM)[22][23] considered in [20], two modulation techniques recommended in IEEE 802.15.6 [3], namely Differential Binary Phase Shift Keying (DBPSK) and Differential Quadrature Phase Shift Keying (DQPSK) are also taken into account. After that, the closed-form expressions of the energy efficiency models of 1-hop, Cooperative, 2-hop and Time diversity communication schemes in multipath fading are explicitly derived for the above-mentioned modulation techniques further. Finally, we evaluate the normalized throughput of all communication schemes with their optimal packet sizes by computer simulations in addition to the average end-to-end (E2E).

According to the numerical results, it is clear that for poor channel conditions (S-D distances exceed a certain threshold), the optimal packet size of our proposed Time Diversity considerably improves the energy efficiency comparing to all communication schemes in inbody channel while our previously proposed 2-hop communication has the best performance in LOS (Line-of-Sight) and NLOS (Non-Line-of-Sight) on-body channels. For Time Diversity communication, the energy efficiency cannot always be improved by increasing the diversity channel since the optimal diversity channel depends on the S-D (Source-Destination) distance. Regarding the performance of modulation techniques, it is obvious that 16QAPM provides the maximum energy efficiency in the in-body channel for all communication schemes excluding Time Diversity communication. On the other hand, BPSK has the best performance in LOS and NLOS on-body channels for all communication schemes.

Next, the simulation results show that Cooperative communication provides the highest normalized throughput in LOS on-body channel. However, our proposed Time Diversity 
communication achieves the best performance in-body channel whereas the previously proposed 2-hop communication does in NLOS on-body channel regardless of S-D distances. In case of the average E2E delay, Cooperative communication is superior to all communication schemes.

The remainder of this paper is organized as follows: Section 2 briefly reviews the closely related prior works. In Section 3, the system model is concisely described. The energy efficiency models of all communication schemes are elaborated in Section 4. The closed-form expressions of the average bit error rate in multipath fading are derived in Section 5. In Section 6, the packet size optimization is investigated. In Section 7, the numerical results of the energy efficiency are demonstrated while in Section 8, the simulation results of the normalized throughput and the average E2E delay are illustrated. Finally, we conclude this paper in Section 9.

\section{RELATED WORK}

Recently, multi-hop and cooperative communications have become of interest to many researchers as effective strategies to improve the energy efficiency and prolong the network lifetime in WBANs. In [11], the energy usage of adding a relay node for multi-hop communication is analyzed. In [12], the relay selection scheme based on residual energy and packet priority for 2-hop communication is proposed. In [13], the authors conduct simulations to study the energy efficiency performance of a multi-hop topology. Moreover, in [14], the energy efficient routing mechanism based on a leach protocol is proposed for multi-hop communications in WSNs. Meanwhile, in [24], the energy efficiency of cooperative communication is investigated, and the outage performance of three transmission schemes, namely 1-hop transmission, 1-relay cooperation and multi-relay cooperation is analyzed. In [25], cooperative transmission with utilization of a diversity technique is proposed in terms of energy efficiency. Furthermore, in [26], cooperative communication adopting the optimal power allocation is evaluated while in [27], cooperative communication employing both the optimal power allocation and the optimal signal-relay location is proposed. However, the issues regarding the optimal packet size have not been discussed in these existing works. In [7] and [8], the energy efficiency models of 1-hop and cooperative communications are proposed to select their appropriate packet size. In [9], the energy efficiency of multi-periodic scheduled access mode as a function of payload size, which relies on the latest drafted IEEE 802.15.6 is derived. In addition, the analysis of power consumption with IEEE 802.15.6 CSMA/CA is carried out to select the optimal packet size in [10]. Nevertheless, it is clear from these works [7]-[10] that the optimal packet size of 2-hop communication has not been investigated yet. Furthermore, in [7] and [8], the multipath fading has not been considered in the case of the inbody channel. Therefore, in [20] and [21] we investigate the energy efficiency of 2-hop communication and then evaluate its performance in terms of the average end-to-end delay. However, the throughput performance has not still been studied.

Diversity technique is also widely used in WBANs to overcome multipath fading, especially when a channel is in deep fading. By transmitting various independently faded copies of the same information to the receiver, the probability that all signal components suffering from deep fading reduces. The overall Signal-to-Noise Ratio (SNR) also increases [28][29]. Typically, diversity technique can be classified into frequency [7], space (receive and transmit diversity)[15]-[18] and time diversity [17]. In [7], frequency technique with the utilization of wideband Direct Sequence-Code Division Multiple Access (DS-CDMA) is used to derive the energy efficiency models in multipath fading in on-body channel. In [15], the transmit diversity with two transmitting antennas being placed on the human body is used in real experiments to deal with the effect of human movements and multipath fading in on-body channel. In [16], the probability of error of $\pi / 4$ DQPSK with two receiving antennas in in-body channel is analyzed. In [17], a two-antenna system design for WBAN in on-body channel is introduced. In addition, the minimum number of antennas and their optimal positions for space diversity in WBAN 
Ultra-Wideband (UWB) is investigated [18]. These works [7][15]-[18] show clearly that frequency and space diversity can mitigate the effect of multipath fading in WBANs, and high diversity gain can be achieved. However, frequency diversity requires additional bandwidths, which may not be compatible with Narrowband on WBANs whereas space diversity may not be implemented in small devices [28], which is a challenging design in WBANs. Thus, we focus on time diversity in this work. In [19], coded pulse position modulation using both time diversity and high dimensional signal constellation over the human abdominal channel and conventional Rayleigh channel is proposed for in-body channel. Nevertheless, to the best of our knowledge, the energy efficiency of time diversity and the optimal packet size has not been explored yet.

\section{SYSTEM MODEL}

In this work, we assume that a relay node (R) is the same type as a source node (S). For example, in on-body channel, both the relay and source nodes are wearable nodes. In addition, the relay node is approximately located in midway between source and destination (D) nodes, since this location provides the maximum energy efficiency [7]. In practice, although the location of the relay node deviates from the midway, its energy efficiency is not so different from the maximum one. Here, we assume that a TDMA-based access mechanism is adopted, and packet collision is not considered.

(R)

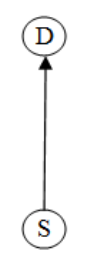

(a) 1-hop

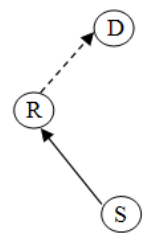

(c) Proposed 2-hop

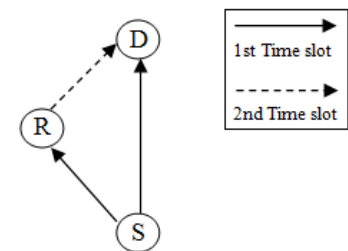

(b) Cooperation
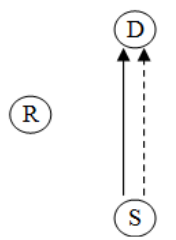

(d) Proposed Time Diversity

Figure 1. Communication schemes under consideration

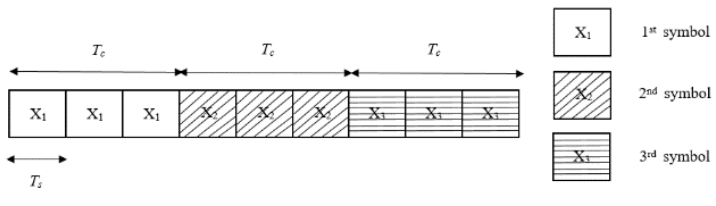

(a) Non-interleaving coding

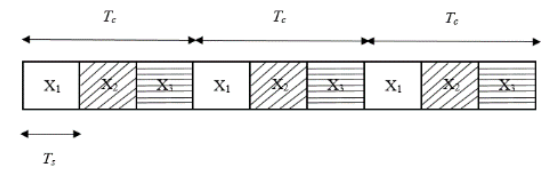

(b) Interleaving coding

Figure 2. Time diversity technique

There are four communication schemes under our consideration, as shown in Figure 1. The transmission procedure of any packets in all communication schemes is carried out as follows: (i) 1-hop communication; the source node directly sends a packet to the destination node in the $1^{\text {st }}$ time slot and so on. (ii) Cooperative communication; the source node broadcasts a packet to the destination and the relay nodes in the $1^{\text {st }}$ time slot. If the transmission from the source to the destination nodes fails, the relay node will retransmit that packet to the destination node in the $2^{\text {nd }}$ time slot. Then, this procedure is repeated for the next packets. (iii) 2-hop communication; the source node sends a packet to the relay node in the $1^{\text {st }}$ time slot and then the relay node forwards that packet to the destination node in the $2^{\text {nd }}$ time slot and so do the next packets. (iv) Time Diversity communication; the source node directly sends $L$ copies of the same packet to the destination node for achieving $L$ diversity channels in $L$ time slots, where $L$ is an integer number.

For Time Diversity communication, repetition coding, which is the simplest technique is adopted. In this technique, information is transmitted in different duration while each duration is 
greater than the coherent time $\left(T_{c}\right)$ of the channel to achieve independent fading. In other words, the gap between consecutive repetition transmissions should be greater than $T_{c}$. In order to do this, the interleaving process is needed, as shown in Figure 2.

In addition, all communication schemes under our consideration adopt ARQ protocol. In this protocol, the destination node sends an ACK or a NACK packet back to the source node depending on whether the destination node can receive the packet correctly or not. In this work, we assume that the transmission of the ACK and NACK packets is error-free because their packet size is very small, and the destination node is generally located outside the human body, so the transmission power can simply be adjusted to accomplish the transmission. In case of Cooperative and 2-hop communications, the destination node broadcasts either the ACK or NACK packets to both the relay and source nodes as a single-hop transmission.

In multipath fading, slow fading channel is considered since it is assumed that user's speed is not high, and only flat fading channel is assumed. In addition, Rician distribution is used to model multipath fading in on-body channel [4] while Rayleigh distribution is adopted in inbody channel since most of the signal components in this channel are transmitted through human's tissues and organs, thus leading to deep fading.

\section{ENERGY EFFICIENCY MODEL}

In this Section, the efficiency models are described in detail.

\subsection{1-hop communication}

In [7] and [8], the energy efficiency model of 1-hop communication $\left(\eta_{1-h o p}\right)$ is proposed and can be expressed as

$$
\eta_{1-h o p}=\frac{\left(1-P E R_{1-h o p}\right)\left(E_{\text {total }}^{1-h o p}\right) s}{E_{\text {total }}^{1-h o p}(S+H)+E_{T x, a c k}+E_{R x, a c k}+E_{\text {active }}}
$$

where $S$ and $H$ represent the payload and header sizes of the transmitted packet in bits, respectively. $P E R_{1-h o p}$ is the packet error rate $(P E R)$ of 1-hop communication (between the source and destination nodes). $E_{\text {total }}^{1-h o p}$, the total energy consumption per bit of 1-hop communication, can be expressed as

$$
E_{\text {total }}^{1-h o p}=E_{T x, \text { process }}+P_{T} / R+E_{R x, \text { process }}
$$

where $P_{T}$ is the transmission power. $R$ is the data rate (bits per second). $E_{T x, \text { process }}$ and $E_{R x, \text { process }}$ are the transmitter and receiver circuit energy consumption for processing data per bit, respectively. It is worth mentioning that $E_{T x, p r o c e s s}$ and $E_{R x \text {,process }}$ are taken into account due to the extremely low transmission power in WBANs. $E_{T x, a c k}$ and $E_{R x, a c k}$ are the energy consumption to transmit and receive the ACK or NACK packet, respectively and can be defined as

$$
\begin{aligned}
& E_{T x, \text { ack }}=\left(E_{T x, \text { process }}+P_{T} / R\right) H_{\text {ack } / \text { nack }} \\
& E_{R x, \text { ack }}=\left(E_{T x, \text { process }}\right) H_{\text {ack } / \text { nack }}
\end{aligned}
$$

where $H_{\text {ack } / \text { nack }}$ is the packet size of the ACK or NACK packet in bits. $E_{\text {active }}$ is the required energy consumption for keeping the transmitter and receiver active. In general, $E_{\text {active }}$ is very small, comparing to the energy consumption for processing and transmitting data and has a 
negligible effect on energy efficiency comparison. Therefore, in this work $E_{\text {active }}$ is a constant value and set to be zero.

\subsection{Cooperative communication}

In [7] and [8], the energy efficiency model of Cooperative communication $\left(\eta_{C o}\right)$ is proposed as well and can be expressed as

$$
\eta_{C o}=\frac{\left(1-P E R_{C o}\right)\left(E_{\text {total }}^{1-h o p}\right) s}{E_{\text {total }}^{C o}(S+H)+E_{T x, a c k}+E_{R x, a c k}+E_{\text {active }}}
$$

where $P E R_{C o}$ is the $P E R$ of Cooperative communication and can be expressed as

$$
P E R_{C o}=P E R_{S D} P E R_{S R}+P E R_{S D}\left(1-P E R_{S R}\right) P E R_{R D}
$$

where $P E R_{S D}, P E R_{S R}$ and $P E R_{R D}$ are the PERs of the links between source and destination, source and relay, and relay and destination nodes, respectively. Equivalently, $P E R_{S D}=P E R_{1-h o p}$. We assume that the packet errors occurring on each link are independent to each other.

$E_{\text {total }}^{C o}$ is the average total energy consumption per bit of Cooperative communication. In order to compute $E_{\text {total }}^{C o}$, three events are considered; the first event is the successful transmission from source to destination nodes in the $1^{\text {st }}$ time slot, which consumes energy $E_{1}$ with probability (1$\left.P E R_{S D}\right)$. Then, $E_{1}$ can be expressed as

$$
E_{1}=E_{T x, \text { process }}+P_{T} / R+2 E_{R x, \text { process }}
$$

The second event is the failure of transmission from source to relay and to destination nodes in the $1^{\text {st }}$ time slot simultaneously, which consumes energy $E_{2}$ with probability $P E R_{S D} P E R_{S R}$. Then, $E_{2}$ can be represented as

$$
E_{2}=E_{T x, \text { process }}+P_{T} / R+2 E_{R x, \text { process }}
$$

The last event is the failure of transmission from source to destination nodes, and the successful transmission from source to relay nodes in the $1^{\text {st }}$ time slot at the same time. This event consumes energy $E_{3}$ with probability $P E R_{S D}\left(1-P E R_{S R}\right)$. Then, $E_{3}$ can be illustrated as

$$
E_{3}=2 E_{T x, \text { process }}+2 P_{T} / R+3 E_{R x, \text { process }}
$$

Therefore, the average total energy consumption per bit of Cooperative communication can be represented as

$$
E_{\text {total }}^{C o}=E_{1}\left(1-P E R_{S D}\right)+E_{2} P E R_{S D} P E R_{S R}+E_{3} P E R_{S D}\left(1-P E R_{S R}\right)
$$

\subsection{Proposed 2-hop communication}

According to our previous work [20], the energy efficiency model of 2-hop communication $\left(\eta_{2-\text { hop }}\right)$ can be expressed as

$$
\eta_{2-\text { hop }}=\frac{\left(1-P E R_{2-h o p}\right)\left(E_{\text {total }}^{1-h o p}\right) S}{E_{\text {total }}^{2-h o p}(S+H)+E_{T x, a c k}+E_{R x, a c k}+E_{\text {active }}}
$$

where $E_{\text {total }}^{2-h o p}$ is the average total energy consumption per bit of 2-hop communication while $P E R_{2-h o p}$ is the PER of 2-hop communication. The packet error in 2-hop communication can 
occur according to two events; the first event is the failure of transmission from source to relay nodes in the $1^{\text {st }}$ time slot. The second event is the successful transmission from source to relay nodes in the $1^{\text {st }}$ time slot, and the failure of transmission from the relay to destination nodes in the $2^{\text {nd }}$ time slot. Consequently, $P E R_{2-h o p}$ can be expressed as

$$
P E R_{2-h o p}=P E R_{S D}+\left(1-P E R_{S R}\right) P E R_{R D}
$$

For computing $E_{\text {total }}^{2-\text { hop }}$, two events are considered; the first event is the successful transmission from source to relay nodes in the $1^{\text {st }}$ time slot, which consumes energy $E_{1}$ with probability (1$\left.P E R_{S R}\right)$. The second event is the failure of transmission from source to relay nodes in the $1^{\text {st }}$ time slot, which consumes energy $E_{2}$ with probability $P E R_{S R}$. Here, $E_{1}$ and $E_{2}$ can be expressed as

$$
E_{1}=2 E_{2}=2\left\{E_{T x, \text { process }}+P_{T} / R+E_{R x, \text { process }}\right\}
$$

Thus, the average total energy consumption per bit of 2-hop can be computed by

$$
E_{\text {total }}^{2-\text { hop }}=E_{1}\left(1-P E R_{S R}\right)+E_{2} P E R_{S R}
$$

\subsection{Proposed time diversity communication}

In this Subsection, we propose the energy efficiency model of Time Diversity communication with the utilization of repetition coding $\left(\eta_{d v}\right)$. It can be given by

$$
\eta_{d v}=\frac{\left(1-P E R_{d v}\right)\left(E_{\text {total }}^{1-h o p}\right) S}{E_{\text {total }}^{\text {dv }}(S+H)+E_{T x, a c k}+E_{R x, a c k}+E_{\text {active }}}
$$

where $E_{t o t a l}^{d v}$ is the total energy consumption per bit of Time Diversity communication. $P E R_{d v}$ is the PER of Time Diversity communication that is equivalent to the PER of the link between the source and destination nodes with Time Diversity technique. For repetition coding, the source node directly transmits $L$ copies of the same packet for achieving $L$ diversity channels to the destination node in $L$ time slots. Consequently, $E_{\text {total }}^{d v}$ can be represented as

$$
E_{\text {total }}^{\text {dv }}=\left(E_{T x, \text { process }}+P_{T} / R+E_{R x, \text { process }}\right) L
$$

\section{The AVerage Bit Error Rate Derivation}

In this Section, we derive the closed-form expressions of the average bit error rate (BER) in multipath fading. Firstly, the packet error rate and the relevant path loss model [30] are considered.

\subsection{The packet error rate in multipath fading}

The PER of each link is defined as the probability which at least one bit in the packet is erroneous. It can be expressed as

$$
P E R=1-\left(1-P_{e}\right)^{S+H}
$$

where $P_{e}$ is the average BER in multipath fading. To compute the average BER, the average instantaneous SNR per bit $(\bar{\gamma})$ is defined as

$$
\bar{\gamma}=\Omega E_{b} / N_{0}
$$

where $\Omega=E\left[\alpha^{2}\right]$ is the mean-square value of the fading amplitude $(\alpha) . E_{b} / N_{0}$ is the SNR per bit and can be given by 


$$
E_{b} / N_{0}(d B)=P_{T}-P L(d)-N_{0}-10 \log (R)
$$

where $N_{0}$ is the AWGN noise power in $\mathrm{dBW} / \mathrm{Hz} . P L(d)$ is the path loss at a distance $d$ in WBAN and can be given by [30]

$$
P L(d)(d B)=P L_{0}+10 n \log \left(d / d_{0}\right)+X_{\sigma}
$$

where $P L_{0}$ represents the path loss at a reference distance $d_{0} . n$ is the path-loss exponent. $X_{\sigma}$ is the shadowing component which is a Gaussian distributed random variable with zero mean and standard deviation in $d B$. All parameters in the path loss model are given in Table 1 [30].

\subsection{The average BER in multipath fading}

In this Subsection, we derive the average BER in multipath fading for 1-hop, 2-hop and Cooperative communications. Under the assumption of flat and slow fading channel, the amplitude attenuation and phase can be considered constant over at least a symbol period. Consequently, the average BER in multipath fading can be computed by averaging the conditional BER for a fixed fading amplitude $(\alpha)$ over the probability density function (PDF) of the fading distribution [28][29] as given in (21) as follow:

$$
P_{e}=\int_{0}^{\infty} P_{b}\left(\gamma_{b}\right) p_{\gamma}\left(\gamma_{b}\right) d \gamma_{b}
$$

where $\gamma_{b}=\alpha^{2} E_{b} / N_{0}$ is the instantaneous SNR per bit. $P_{b}\left(\gamma_{b}\right)$ is the BER conditioned on a fixed $\gamma_{b}$ (the conditional BER). It is equivalent to the BER over AWGN channel, where $E_{b} / N_{0}$ is set to be $\gamma_{b} . p_{\gamma}\left(\gamma_{b}\right)$ is the PDF of $\gamma_{b}$ corresponding to the fading distribution.

In this work, we consider four types of modulation techniques, namely BPSK, 16QAPM, DBPSK and DQPSK. According to [22], the conditional BER of 16QAPM can be expressed as shown in (22).

$$
\begin{aligned}
P_{b, 16 Q A P M}\left(\gamma_{b}\right) & =\frac{1}{4}\left[\frac{5}{2} Q\left(\sqrt{4 \gamma_{b} / 3}-\frac{1}{3} Q^{2} \sqrt{4 \gamma_{b} / 3}\right)\right] \\
Q(x) & =\frac{1}{\pi} \int_{0}^{\pi / 2} \exp \left(\frac{-x^{2}}{2 \sin ^{2} \theta}\right) d \theta, x \geq 0 \\
Q^{2}(x) & =\frac{1}{\pi} \int_{0}^{\pi / 4} \exp \left(\frac{-x^{2}}{2 \sin ^{2} \theta}\right) d \theta, x \geq 0
\end{aligned}
$$

By using alternative expressions of Q-function and the square of Q-function [29] as given in (23) and (24), the conditional BER of 16QAPM can be rewritten in the form of exponential function of $\gamma_{b}$ as

$$
P_{b, 16 Q A P M}\left(\gamma_{b}\right)=\frac{5}{8 \pi} \int_{0}^{\pi / 2} \exp \left(\frac{-2 \gamma_{b}}{3 \sin ^{2} \theta}\right) d \theta-\frac{1}{12 \pi} \int_{0}^{\pi / 4} \exp \left(\frac{-2 \gamma_{b}}{3 \sin ^{2} \theta}\right) d \theta
$$

Then, by substituting (25) into (21), we can represent the average BER of 16QAPM in terms of Moment Generating Function (MGF) as

$$
P_{e, 16 Q A P M}=\frac{5}{8 \pi} \int_{0}^{\pi / 2} M_{\gamma}\left(\frac{-2}{3 \sin ^{2} \theta}\right) d \theta-\frac{1}{12 \pi} \int_{0}^{\pi / 4} M_{\gamma}\left(\frac{-2}{3 \sin ^{2} \theta}\right) d \theta
$$

where the MGF is defined as $M_{\gamma}(s)=\int_{0}^{\infty} p_{\gamma}\left(\gamma_{b}\right) e^{s \gamma_{b}} d \gamma_{b}$. In the Rayleigh fading channel, the average BER of 16QAPM can be derived further and expressed in a closed-form as (See Appendix A.1 for detailed proof) 
International Journal of Computer Networks \& Communications (IJCNC) Vol.10, No.5, September 2018

$$
P_{e, 16 Q A P M}=\frac{5}{16}\left(1-\sqrt{\frac{2 \bar{\gamma}}{3+2 \bar{\gamma}}}\right)-\frac{1}{48}\left(1-\frac{4}{\pi} \sqrt{\frac{2 \bar{\gamma}}{3+2 \bar{\gamma}}}\left[\tan ^{-1}\left(\sqrt{\frac{3+2 \bar{\gamma}}{2 \bar{\gamma}}}\right)\right]\right)
$$

where $\bar{\gamma}$ is the average instantaneous SNR per bit as shown in (18). In the Rician fading channel, by putting the MGF of the Rician distribution into (26), we obtain the closed-form expression of the average BER of 16QAPM with finite integral, where $K$ is the Rician factor.

In [29], the average BERs of BPSK, DBPSK and DQPSK over the fading channel are derived in terms of MGF. Furthermore, in the Rayleigh fading channel, the average BER of BPSK and DQPSK can further be simplified and expressed in closed-forms as illustrated in (28) and (29), respectively.

$$
\begin{aligned}
P_{e, B P S K} & =\frac{1}{2}\left(1-\sqrt{\frac{\bar{\gamma}}{1+\bar{\gamma}}}\right) \\
P_{e, D Q P S K} & =\frac{1}{2}\left(1-\frac{\sqrt{2} \bar{\gamma}}{\sqrt{1+4 \bar{\gamma}+2 \bar{\gamma}^{2}}}\right)
\end{aligned}
$$

\subsection{The average BER with $L$ diversity channels}

In this Subsection, the average BER for Time Diversity communication in multipath fading is derived. We assume that there are $L$ diversity channels that contain the same information with independent fading amplitudes. Also, multipath fading among $L$ diversity channels is assumed to be independent identically distributed (i.i.d); fading distributions are the same types and have the same fading parameters. For coherent detection, Maximal Ratio Combining (MRC)[29] is used as a technique to efficiently combine $L$ independently faded copies of the same packet at the receiver. Since MRC requires knowledge of all fading parameters, it is the optimal scheme. In the case of differentially coherent detection, Equal-Gain Combining (EGC)[29], which is the suboptimal scheme, is utilized.

The main idea to derive the average BER with $L$ diversity channels is to find the BER conditioned on a fixed set of $\left\{\alpha_{c} \mid c \in 1,2,3, \ldots, L\right\}$ and then average it over the PDF of $\left\{\alpha_{c}\right\}$. Consequently, the average BER as shown in (21) can be rewritten as

$$
P_{e}=\int_{0}^{\infty} P_{b}\left(\gamma_{t}\right) p\left(\gamma_{t}\right) d \gamma_{t}
$$

where $\gamma_{t}$ is the total instantaneous SNR per bit. For equally likely transmitted symbols, $\gamma_{t}$ can be computed [28] by

$$
\gamma_{t}=\sum_{C=1}^{L} \gamma_{C}
$$

where $\gamma_{c}=\alpha_{c}^{2} E_{b} / N_{0}$ is the instantaneous SNR per bit on $c^{\text {th }}$ channel. $P_{b}\left(\gamma_{t}\right)$ is the conditional BER for a fixed set of $\left\{\gamma_{c}\right\}$. It is equivalent to the BER over AWGN multichannel, where the total SNR is set to be $\gamma_{t} \cdot P_{\gamma}\left(\gamma_{t}\right)$ is the PDF of $\gamma_{t}$. Without any loss in performance due to coherent detection, the conditional BER of 16QAPM for a fixed set of $\left\{\gamma_{c}\right\}$, can be expressed as

$$
P_{b, 16 Q A P M}\left(\gamma_{t}\right)=\frac{1}{4}\left[\frac{5}{2} Q\left(\sqrt{4 \gamma_{t} / 3}\right)-\frac{1}{3} Q^{2}\left(\sqrt{4 \gamma_{t} / 3}\right)\right]
$$

Similar to Subsection 5.2, (32) can be rewritten as

$$
P_{b, 16 Q A P M}\left(\gamma_{t}\right)=\frac{5}{8 \pi} \int_{0}^{\frac{\pi}{2}} \prod_{c=1}^{L} \exp \left(\frac{-2 \gamma_{c}}{3 \sin ^{2} \theta}\right) d \theta-\frac{1}{12 \pi} \int_{0}^{\pi / 4} \prod_{c=1}^{L} \exp \left(\frac{-2 \gamma_{c}}{3 \sin ^{2} \theta}\right) d \theta
$$


International Journal of Computer Networks \& Communications (IJCNC) Vol.10, No.5, September 2018

By substituting (33) into (30) and due to the i.i.d fading distribution, the average BER of 16QAPM can be written as

$$
P_{e, 16 Q A P M}=\frac{5}{8 \pi} \int_{0}^{\pi / 2}\left[M_{\gamma}\left(\frac{-2}{3 \sin ^{2} \theta}\right)\right]^{L} d \theta-\frac{1}{12 \pi} \int_{0}^{\pi / 4}\left[M_{\gamma}\left(\frac{-2}{3 \sin ^{2} \theta}\right)\right]^{L} d \theta
$$

In the Rayleigh fading channel, the average BER of 16QAPM with $L$ diversity channels can be simplified to (See Appendix A.2 for detailed proof)

$$
\begin{aligned}
& P_{e, 16 Q A P M}=\frac{5}{8}\left(\frac{1-\mu_{y}}{2}\right)^{L} \sum_{c=0}^{L-1}\left(\begin{array}{c}
L-1+c \\
c
\end{array}\right)\left(\frac{1+\mu_{y}}{2}\right)^{c}+\frac{\mu_{y}}{12 \pi}\left(\frac{\pi}{2}-\tan ^{-1} \mu_{y}\right) \sum_{c=0}^{L-1}\left(\begin{array}{c}
2 c \\
c
\end{array}\right)\left(\frac{1}{4(1+(2 / 3) \bar{\gamma})}\right)^{c} \\
& -\frac{1}{48}-\frac{\mu_{y}}{12 \pi} \sin \left(\tan ^{-1} \mu_{y}\right) \sum_{c=1}^{L-1} \sum_{i=1}^{c} \frac{T_{i c}}{(1+(2 / 3) \bar{\gamma})^{c}}\left[\cos \left(\tan ^{-1} \mu_{y}\right)\right]^{2(c-i)+1} \\
& \text { where } \\
& \qquad \mu_{y}=\sqrt{\frac{2 \bar{\gamma}}{3+2 \bar{\gamma}}} \\
& \text { and } \\
& \qquad T_{i c}=\frac{\left(\begin{array}{c}
2 c \\
c
\end{array}\right)}{\left(\begin{array}{c}
(c-i) \\
c-\mathrm{i}
\end{array}\right) 4^{i}[2(c-i)+1]}
\end{aligned}
$$

In [29], the average BER of BPSK with $L$ diversity channels over the fading channel in terms of MGF can be expressed as

$$
P_{e, B P S K}=\frac{1}{\pi} \int_{0}^{\pi / 2}\left[M_{\gamma}\left(\frac{-1}{\sin ^{2} \theta}\right)\right]^{L} d \theta
$$

In addition, the average BERs of DBPSK and DQPSK have identical expressions and can be represented as follow:

$$
P_{e, D B P S K}=P_{e, D Q P S K}=\frac{1}{2^{2 L} \pi} \int_{-\pi}^{\pi} \frac{f(L ; \varepsilon ; \theta)}{1+2 \varepsilon \sin \theta+\varepsilon^{2}}\left[M_{\gamma}\left(-\frac{a^{2}+b^{2}+2 a b \sin \theta}{2}\right)\right]_{a}^{L} d \theta,
$$

where $a=0$ and $b=\sqrt{2}$ for DBPSK while $a=\sqrt{2-\sqrt{2}}$ and $b=\sqrt{2+\sqrt{2}}$ for DQPSK. $f(L ; \varepsilon ; \theta)$ is defined as

$$
\begin{gathered}
f(L ; \varepsilon ; \theta)=\sum_{c=1}^{L}\left(\begin{array}{c}
2 L-1 \\
L-c
\end{array}\right)\left\{\left(\varepsilon^{-c+1}-\varepsilon^{c+1}\right) \cos [(c-1)(\theta+\pi / 2)]\right. \\
\left.-\left(\varepsilon^{-c+2}-\varepsilon^{c}\right) \cos [c(\theta+\pi / 2)]\right\}
\end{gathered}
$$

Furthermore, in the Rayleigh fading channel, the average BER of BPSK with $L$ diversity channels can be further simplified and given by [29] as follow:

$$
P_{e, B P S K}=\left(\frac{1-\mu_{x}}{2}\right)^{L} \sum_{c=0}^{L-1}\left(\begin{array}{c}
L-1+c \\
c
\end{array}\right)\left(\frac{1+\mu_{x}}{2}\right)^{c}
$$

where

$$
\mu_{x}=\sqrt{\frac{\bar{\gamma}}{1+\bar{\gamma}}}
$$

and the average BER of DBPSK with $L$ diversity channels can be expressed in a closed-form [28] as

$$
P_{e, D B P S K}=\frac{1}{2^{2 L-1}(L-1) !(1+\bar{\gamma})^{L}} \sum_{c=0}^{L-1} b_{c}(L-1+c) !\left(\frac{\bar{\gamma}}{1+\bar{\gamma}}\right)^{c}
$$


International Journal of Computer Networks \& Communications (IJCNC) Vol.10, No.5, September 2018

where

$$
b_{c}=\frac{1}{c !} \sum_{k=0}^{L-1-c}\left(\begin{array}{c}
2 L-1 \\
\mathrm{k}
\end{array}\right)
$$

In the Rician fading channel, the closed-form expressions of the average BERs of BPSK, 16QAPM, DBPSK and DQPSK with $L$ diversity channels can be obtained with finite integral.

\section{Packet Size Optimization}

An optimal packet size is defined as a payload size of a packet which maximizes energy efficiency. Let $S_{1-h o p}^{o p t}, S_{2-h o p}^{O p t}, S_{C o}^{O p t}$ and $S_{d v}^{O p t}$ be the optimal payload sizes of 1-hop, 2-hop, Cooperative and Time Diversity communications, respectively. They can be obtained by taking $\frac{\partial \eta}{\partial s}=0$ given that $\eta$ is a concave function, where $\eta$ is the energy efficiency model of 1-hop, 2hop, Cooperative and Time Diversity communications.

For 1-hop communication, the closed-form expression of the optimal payload size can be obtained as follow:

$$
S_{1-h o p}^{\text {opt }}=-\frac{x}{2}+\frac{1}{2} \sqrt{x^{2}-\frac{4 x}{\ln \left(1-P_{e}\right)}}
$$

where $x=H+H_{\text {ack } / \text { nack }}$; $H$ denotes the header size, and $H_{\text {ack } / \text { nack }}$ is the packet size of ACK or NACK packet. In case of Time Diversity communication, the optimal payload size can be expressed in a closed-form as

$$
S_{d v}^{O p t}=-\frac{y}{2}+\frac{1}{2} \sqrt{y^{2}-\frac{4 y}{\ln \left(1-P_{e}\right)}}
$$

where $y=\left(H L+H_{\text {ack } / \text { nack }}\right) / L$. The closed-form expressions of $S_{2-h o p}^{O p t}$ and $S_{C o}^{O p t}$ cannot be obtained simply. However, we find that $S_{2-h o p}^{O p t}$ and $S_{C o}^{O p t}$ exist in the numerical result section, and they can be obtained simply by using optimization programming.

Table 1. Parameter values

\begin{tabular}{|l|l|}
\hline Parameter & Value \\
\hline$S_{\text {max }} / \mathrm{H} / H_{\text {ack } / \text { nack }}$ & $2000 / 80 / 64$ bits \\
\hline$P_{T}$ & $-10 \mathrm{dBm}$ \\
\hline$N_{0}$ & $-180 \mathrm{dBW} / \mathrm{Hz}$ \\
\hline LOS on-body & $d_{0}: 10 \mathrm{~cm}, n: 3.11, \sigma: 6.1 \mathrm{~dB}$, \\
& $P L_{0}: 35.2 \mathrm{~dB}, f: 2.45 \mathrm{GHz}$ \\
\hline NLOS on-body & $d_{0}: 10 \mathrm{~cm}, n: 5.9, \sigma: 5.0 \mathrm{~dB}$, \\
& $P L_{0}: 48.4 \mathrm{~dB}, f: 3.1 \mathrm{GHz}$ \\
\hline In-body & $d_{0}: 5 \mathrm{~cm}, n: 4.22, \sigma: 6.81 \mathrm{~dB}$, \\
& $P L_{0}: 49.81 \mathrm{~dB}, f: 402-405 \mathrm{MHz}$ \\
\hline Data rate $(\mathrm{R})$ & On-body/In-body: $2 / 1 \mathrm{Mbps}$ \\
\hline$E_{T x, \text { process }}=E_{R x, \text { process }}$ & On-body/In-body: $11.25 / 18.75 \mathrm{~nJ} / \mathrm{bit}$ \\
\hline
\end{tabular}

Table 2. Fading parameters

\begin{tabular}{|c|c|c|c|}
\hline \multirow{2}{*}{ Channel model } & \multicolumn{2}{|c|}{ Rician[4] } & Rayleigh \\
\cline { 2 - 4 } & $K(d B)$ & $\Omega$ & $\Omega$ \\
\hline LOS on-body & 24.7 & 1 & - \\
\hline NLOS on-body & 9.3 & 1.03 & - \\
\hline In-body & - & - & 2 \\
\hline
\end{tabular}




\section{NUMERICAL RESULTS AND DISCUSSION}

In this Section, the energy efficiency of the proposed 2-hop and Time Diversity are evaluated and then compared to the existing 1-hop and Cooperative communications in multipath fading using the numerical calculation. All parameters used are given in Table 1 and 2.

\subsection{The effect of multipath fading}

First, we investigate the impact of multipath fading on energy efficiency in all WBAN channels. In NLOS on-body channel, as shown in Figure 3(a), multipath fading affects energy efficiency obviously. The maximum energy efficiency of BPSK and 16QAPM reduce from non-fading to fading cases approximately $54 \%$ and $58 \%$, respectively. Moreover, the payload size in fading case has a larger impact on energy efficiency than the one in the non-fading case for the same S$\mathrm{D}$ distance. The energy efficiency with fading case drops sharply when the payload size deviates from the optimal one. In case of the larger payload size, energy efficiency reduces due to the high probability of packet error while for the smaller payload size, energy efficiency reduces because of the large proportion of control overhead. However, due to low signal scattering, the impact of multipath fading on energy efficiency in LOS on-body channel is quite small.

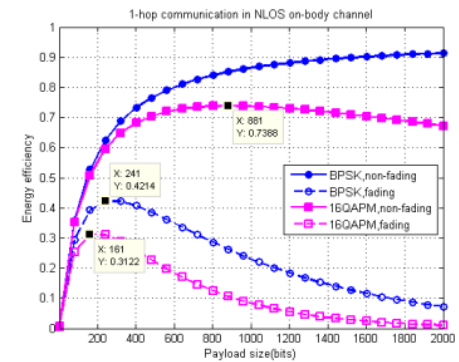

(a) NLOS on-body channel at S-D distance of $21 \mathrm{~cm}$

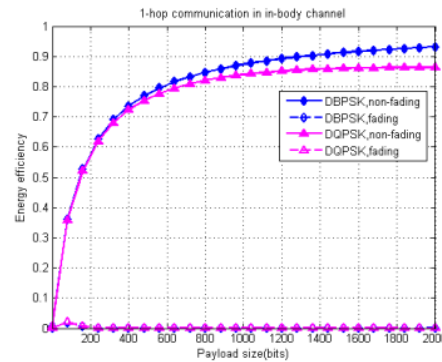

(b) In-body channel at S-D distance of $14 \mathrm{~cm}$

Figure 3. Energy efficiency Vs. payload size for 1-hop communication

In case of the in-body channel, as shown in Figure 3(b), the energy efficiency with fading case is extremely poor comparing to the non-fading case because of high signal scattering. In addition, the energy efficiency of DBPSK and DQPSK for 1-hop communication approach zero in multipath fading when the S-D distance is greater than approximately $14 \mathrm{~cm}$ due to wasteful energy consumption.

\subsection{The energy efficiency of Time Diversity communication with various $L$}

In this Subsection, the impact of various $L$ diversity channels on energy efficiency in multipath fading is investigated. All modulation techniques under consideration in this work are evaluated. However, only the result of DQPSK is illustrated here because of the same trend of the results obtained.

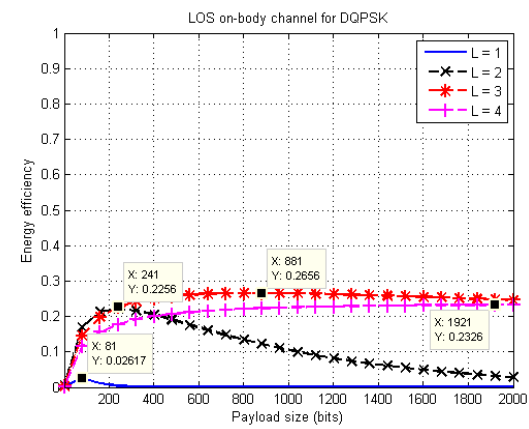

Figure 4. Energy efficiency Vs. payload size for Time Diversity communication for DQPSK for LOS on-body channel at S-D distance of $140 \mathrm{~cm}$ 
The energy efficiency of Time Diversity communication with various $L$ diversity channels in LOS on-body channel is plotted in Figure 4. It is apparent that increasing the value of $L$ does not always improve energy efficiency. According to the results in Figure 4, the optimal value of $L$ is 3 with the maximum energy efficiency of $26.5 \%$. However, when the value of $L$ increases to 4 , the maximum energy efficiency drops to $22.5 \%$. This is because increasing value of $L$ requires a large amount of energy consumption without significant improvement of the successful transmission, when the transmission is error-free, or the PER approaches zero.

\subsection{The energy efficiency of four communication schemes in multipath fading}

In this Subsection, we evaluate and compare the impact of the optimal packet size on the energy efficiency of 1-hop, 2-hop, Cooperative and Time Diversity communications. For Time Diversity communication, the optimal diversity channel $\left(L_{O p t}\right)$ is considered as well as the diversity channel of $2(L=2)$ since the high value of $L$ leads to higher delay. We evaluate all modulation techniques under consideration in this work. However, only the result of DBPSK is illustrated here because of the same trend of results obtained.

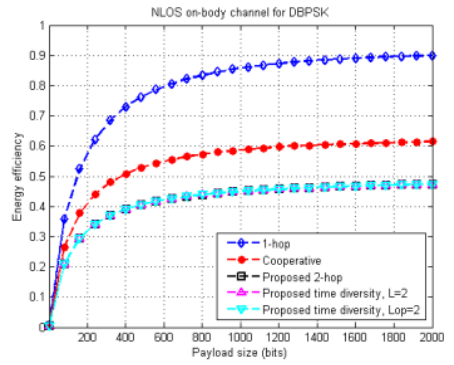

(a) S-D distance of $14 \mathrm{~cm}$

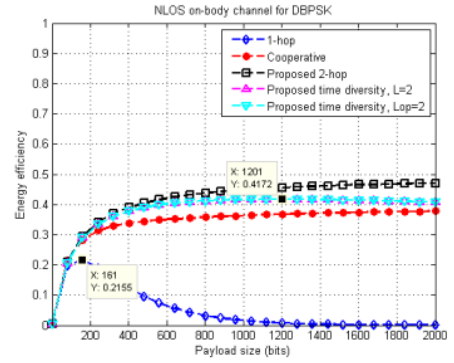

(b) S-D distance of $21 \mathrm{~cm}$

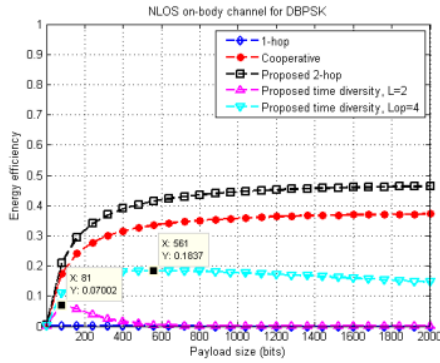

(c) S-D distance of $25 \mathrm{~cm}$

Figure 5. Energy efficiency Vs. payload size in NLOS on-body channel for DBPSK

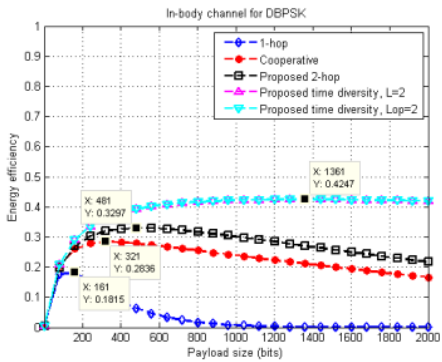

(a) S-D distance of $10 \mathrm{~cm}$

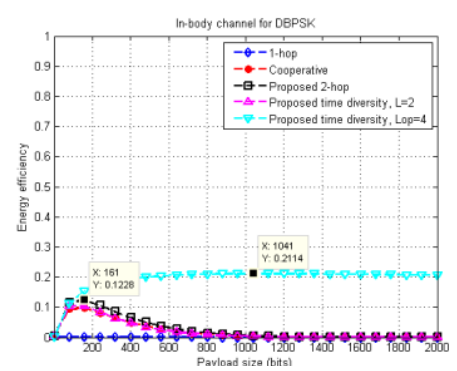

(b) S-D distance of $17 \mathrm{~cm}$

Figure 6. Energy efficiency Vs. payload size in in-body channel for DBPSK

First, we consider LOS and NLOS on-body channels. The energy efficiency versus payload size in NLOS on-body channel in multipath fading for various S-D distances is plotted in Figure 5. For good channel conditions, as shown in Figure 5(a), the optimal payload sizes of 1-hop and Cooperative provide better energy efficiency than that of our proposed 2-hop communication. This is because at this S-D distance, the probability of direct successful transmission is very high, so 1-hop and Cooperative consume less energy than 2-hop communication. Also, they are better than our proposed Time Diversity communication for both $L=2$ and $L_{O p t}$ because the probability of successful transmission of Time Diversity does not significantly increase in case of low signal scattering, and it consumes much higher energy. 
However, when the channel is poor (S-D distances exceed $21 \mathrm{~cm}$ ), as shown in Figure 5(b), the optimal payload sizes of our proposed 2-hop and Time Diversity achieve better energy efficiency than those of 1-hop and Cooperative communications. The maximum energy efficiency of 2-hop is better than those of 1-hop and Cooperative communications approximately $114 \%$ and $27 \%$, respectively. While in the case of Time Diversity, it is better than 1-hop and Cooperative communications roughly $94 \%$ and $14 \%$, respectively. The 2-hop and Time Diversity are better than 1-hop communication because the energy consumption of 1hop communication is very likely to be wasteful due to the low probability of direct successful transmission. Also, they outperform Cooperative because Cooperative communication consumes higher energy for both direct and relay transmissions.

Table 3. Threshold distance

\begin{tabular}{|l|c|c|c|c|c|c|c|c|}
\hline \multirow{2}{*}{ Channel model } & \multicolumn{2}{|c|}{ BPSK } & \multicolumn{2}{c|}{ 16QAPM } & \multicolumn{2}{c|}{ DBPSK } & \multicolumn{2}{c|}{ DQPSK } \\
\cline { 2 - 9 } & $\begin{array}{c}d_{\text {threshold }} \\
(\mathrm{cm})\end{array}$ & $\begin{array}{c}r_{d} \\
(\mathrm{~cm})\end{array}$ & $\begin{array}{c}d_{\text {threshold }} \\
(\mathrm{cm})\end{array}$ & $\begin{array}{c}r_{d} \\
(\mathrm{~cm})\end{array}$ & $\begin{array}{c}d_{\text {threshold }} \\
(\mathrm{cm})\end{array}$ & $\begin{array}{c}r_{d} \\
(\mathrm{~cm})\end{array}$ & $\begin{array}{c}d_{\text {threshold }} \\
(\mathrm{cm})\end{array}$ & $\begin{array}{c}r_{d} \\
(\mathrm{~cm})\end{array}$ \\
\hline LOS on-body & 133 & - & 120 & - & 122 & - & 111 & - \\
\hline NLOS on-body & 21 & - & 20 & - & 20 & - & 19 & - \\
\hline In-body & 21 & $9-20$ & 19 & $9-18$ & 17 & $8-16$ & 18 & $7-17$ \\
\hline
\end{tabular}

Note that diversity channel $(L)$ is equal to 2 for Time Diversity communication

Table 4. Comparison of four modulation techniques

\begin{tabular}{|l|c|c|c|c|c|c|}
\hline \multirow{3}{*}{ Modulation } & \multicolumn{2}{|c|}{ LOS on-body } & \multicolumn{2}{c|}{ NLOS on-body } & \multicolumn{2}{c|}{ In-body } \\
\cline { 2 - 7 } & $\begin{array}{c}\text { 1-hop, Co } \\
\text { and 2-hop }\end{array}$ & $\begin{array}{c}\text { Time } \\
\text { Diversity }\end{array}$ & $\begin{array}{c}\text { 1-hop, Co } \\
\text { and 2-hop }\end{array}$ & $\begin{array}{c}\text { Time } \\
\text { Diversity }\end{array}$ & $\begin{array}{c}\text { 1-hop, Co } \\
\text { and 2-hop }\end{array}$ & $\begin{array}{c}\text { Time } \\
\text { Diversity }\end{array}$ \\
\hline BPSK & 1 & 1 & 1 & 1 & 2 & 1 \\
\hline 16QAPM & 3 & 2 & 2 & 2 & 1 & 2 \\
\hline DBPSK & 2 & 3 & 3 & 3 & 3 & 3 \\
\hline DQPSK & 4 & 4 & 4 & 4 & 3 & 3 \\
\hline
\end{tabular}

Note that number 1, 2, 3 and 4 indicates the first, second, third and fourth highest energy efficiency, respectively.

For extremely poor channel conditions, Time Diversity with $L=2$ and even the optimal $L\left(L_{O p t}\right.$ $=4$ ) is not sufficient anymore while Cooperative is still operable by using relay communication. As a result, Cooperative outperforms Time Diversity communication again, as shown in Figure 5(c). In addition, it is shown clearly in Figure 5 that 2-hop is always superior to Time Diversity communication with any diversity channel in LOS and NLOS on-body channels regardless of SD distances, since Time Diversity does not effectively operate in low signal scattering situation. This implies that for very large S-D distances, to decrease transmission distance provides better energy efficiency than to transmit any independently faded copies of the same packet to the receiver in these channels. The summarization of the approximated threshold distance $\left(d_{\text {threshold }}\right)$, where 2-hop communication has the best performance in LOS and NLOS on-body channels is given in Table 3 .

Next, we consider in-body channel, as shown in Figure 6. The optimal payload size of our proposed Time Diversity communication can provide the maximum energy efficiency, as shown in Figure 6(a) since the impact of multipath fading is very high in the in-body channel. In addition, for extremely poor channel conditions as shown in Figure 6(b), although Time Diversity communication with $L=2$ is inferior to 2-hop communication, it can achieve the maximum energy efficiency by using the optimal $L\left(L_{O p t}=4\right)$. We summarize the approximated range of distances $\left(r_{d}\right)$ where Time Diversity communication with $L=2$ has the 
best performance, as well as the $d_{\text {threshold }}$ in the in-body channel in Table 3 since $L=2$ is more practical for strict delay applications.

In this work, we also investigate the energy efficiency of the four modulation techniques in each communication scheme, and their performance is compared together in Table 4. It is found that 16QAPM provides the highest energy efficiency in in-body channel for 1-hop, Cooperative and 2-hop communications whereas BPSK has the best performance among the rest. Since the energy efficiency is inversely proportional to the average BER, some mathematical proofs can be obtained due to the existence of the closed-form expressions of the average BER (See Appendix B).

\section{SimUlation RESUltS AND DisCUSSION}

In this Section, we simulate and compare the normalized throughput and the average E2E delay of 1-hop, 2-hop, Cooperative and Time Diversity communications adopting their optimal packet sizes. All simulations are implemented using MATLAB version R2010a. Here, only one S-D pair is considered. The ARQ protocol utilizing stop-and-wait mechanism is adopted. In this mechanism, when the transmission is successful, the destination node sends an ACK packet back to the source node for requesting a subsequent packet. Also, the retransmission of any packet is made only when the destination node sends a NACK packet back to the source node. For Time Diversity communication, $L$ is set to be 2 . All parameters used for simulation are given in Table 1 and 2.

\subsection{Normalized throughput Vs. S-D distance}

The normalized throughput is defined as the ratio of the number of successfully received bits of the packets using the optimal packet size and the number of transmitted bits using the maximum packet size. The number of transmitted packets is set to be 1,000 packets for all communication schemes, and their payload size is selected based on the maximum energy efficiency.

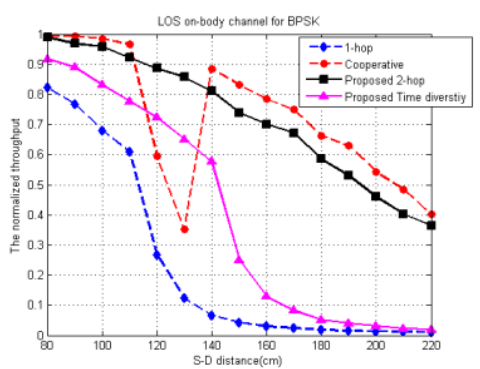

(a) LOS on-body channel

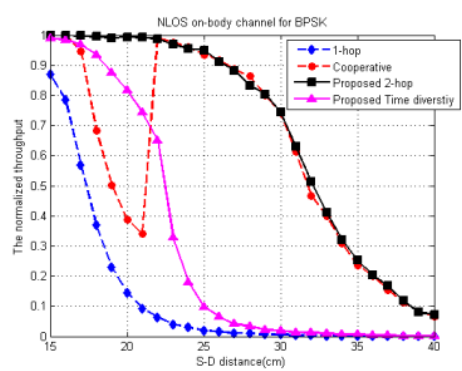

(b) NLOS on-body channel

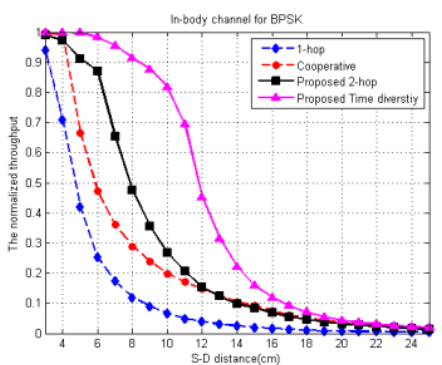

(c) In-body channel

Figure 7. The normalized throughput Vs. the S-D distance for BPSK

The simulation results of BPSK are plotted in Figure 7. In LOS on-body channel, as shown in Figure 7(a), Cooperative communication adopting its optimal payload size provides the highest normalized throughput for almost the whole S-D distances. Nevertheless, when the S-D distance is around 115 to $135 \mathrm{~cm}$, our proposed 2-hop and Time Diversity communications achieve higher normalized throughput because in Cooperative communication, the packet size is reduced to fulfil packet transmission within the $1^{\text {st }}$ time slot to keep the energy efficiency high. Furthermore, in the case of NLOS on-body channel, as shown in Figure 7(b), our proposed 2hop communication provides the highest normalized throughput for the entire S-D distances. As mentioned previously, our proposed Time Diversity communication effectively performs in the 
environment with the high impact of multipath fading. Therefore, it provides the highest normalized throughput in in-body channel, as shown in Figure 7(c).

\subsection{Average End-to-End delay Vs. S-D distance}

In this Subsection, we simulate the average E2E delay of a S-D pair for all communication schemes which is defined as the average time spent on transmitting all amount of data to the destination node. In LOS and NLOS on-body channels, the number of transmitted bits is 200 kbits which are enough to support on-body information such as blood pressure and heart rate. The modulation technique used in these channels is DBPSK. In case of in-body channel, the number of transmitted bits is 6.4 Mbits (approximately equivalent to 5 still images) to support image information with DQPSK. The detail of delay analysis can be found in [21].

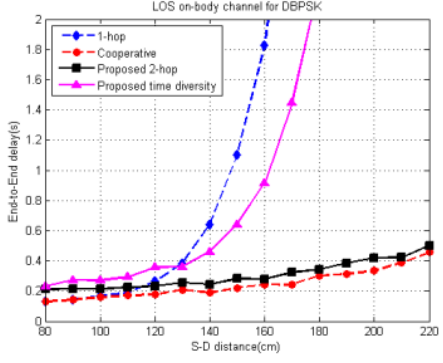

(a) LOS on-body channel

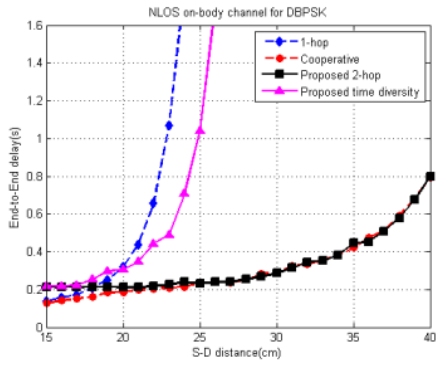

(b) NLOS on-body channel

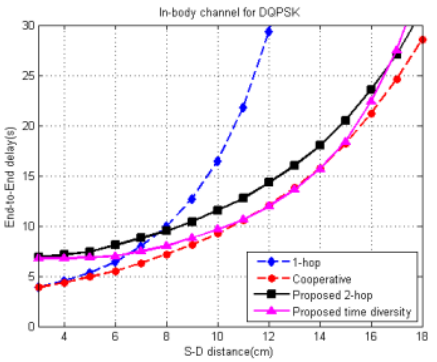

(c) In-body channel

Figure 8. The average E2E delay Vs. the S-D distance for DQPSK

The simulation results in all WBAN channels are shown in Figure 8. It is clear that Cooperative communication provides the lowest average E2E delay for the entire S-D distances in all WBAN channels. Nevertheless, when the S-D distance exceeds a certain value, the average E2E delay of Cooperative and our proposed 2-hop communications are close to each other. Moreover, in in-body channel at the S-D distance between 11 and $15 \mathrm{~cm}$, our proposed Time Diversity approximately has the same average E2E delay as Cooperative communication.

\section{CONCLUSION}

In our work, we investigated the energy efficiency of Time Diversity communication in multipath fading for several modulation techniques. The general closed-form expression of the energy efficiency model and the optimal packet size in Rician and Rayleigh fading channels were also derived. Furthermore, the normalized throughput and the average E2E delay of 1-hop, Cooperative, 2-hop and Time Diversity communication schemes adopting their optimal packet sizes were simulated and compared. The results demonstrate that our proposed Time diversity communication with the optimal number of diversity channels $\left(L_{O p t}\right)$ achieves the highest energy efficiency for poor channel conditions and the normalized throughput for the entire S-D distances in in-body channel. On the other hand, 2-hop communication provides the best performance in terms of the energy efficiency and the normalized throughput in LOS and NLOS on-body channels while Cooperative communication outperforms all communication schemes in terms of the average end-to-end delay. However, it is important to mention that our proposed Time Diversity does not require any relay nodes that might bring inconvenience to human users.

In our future work, WBAN adopting the multi-input multi-output (MIMO) technology to achieve high data rate transmission will be investigated. Furthermore, the impact of channel coding on energy efficiency will be explored. 
International Journal of Computer Networks \& Communications (IJCNC) Vol.10, No.5, September 2018

\section{REFERENCES}

[1] N. F. Timmonsand W. G. Scanlon, "An adaptive energy efficient MAC protocol for the medical body area network,"1st International Conference on Wireless Communication, Vehicular Technology, Information Theory and Aerospace \& Electronic Systems Technology, Aalborg, 2009, pp. 587-593.

[2] J. Shi, Y Takagi, D. Anzai and J. Wang, "Performance evaluation and link Budget analysis on dualmode communication system in Body Area Networks."IEICE TRANS. COMMUN., vol. E97-B, no.6.2014.

[3] The IEEE 802.15.6. IEEE 802.15 TG6. http://www.ieee802.org/15/ pub/TG6/html.

[4] S. L. Cotton and W. G. Scanlon, "A statistical analysis of indoor multipath fading for a narrowband wireless body area network,"IEEE 17th International Symposium on Personal, Indoor and Mobile Radio Communications, Helsinki, 2006, pp. 1-5.

[5] Y. I. Nechayev, P. S. Hall, I. Khan and C. C. Constantinou, "Wireless channels and antennas for body-area networks," 7th International Conference on Wireless On-demand Network Systems and Services (WONS), Kranjska Gora, 2010, pp. 137-144.

[6] Y. Fang, Z. Wu, L. Jiang and Y. Sun, "Performance evaluation of RAKE receiver for ultra-wideband wireless body area network communication,"12th International Conference on Signal Processing (ICSP), Hangzhou, 2014, pp. 2257-2261.

[7] K. S. Deepak and A. V. Babu, "Packet size optimization for energy efficient cooperative Wireless Body Area Networks,”Annual IEEE India Conference (INDICON), Kochi, 2012, pp. 736-741

[8] K. S. Deepak amd A. V. Babu, "Improving energy efficiency of incremental relay based cooperative communications in wireless body area networks: ENERGY EFFICIENCY OF COOPERATIVE COMMUNICATIONS IN WBANs,’International Journal of Communication Systems, 2013.

[9] K. S. Deepak and A. V. Babu, "Optimal packet size for energy efficient WBAN under m-periodic scheduled access mode,"20th National Conference on Communications (NCC), Kanpur, 2014, pp. 16.

[10] P. T. Hiep and N. Huy Hoang, "Payload and power consumption analysis of IEEE802.15.6 based WBAN with CSMA/CA,"International Conference on Advanced Technologies for Communications (ATC 2014), Hanoi, 2014, pp. 500-505.

[11] A. Ehyaie, M. Hashemi and P. Khadivi, "Using relay network to increase life time in wireless body area sensor networks,"IEEE International Symposium on a World of Wireless, Mobile and Multimedia Networks \& Workshops, Kos, 2009, pp. 1-6.

[12] R. Cabacas, H. Yang and I. H. Ra, "Energy-efficient two-hop transmission prioritization scheme for wireless body area networks,"Joint 7th International Conference on Soft Computing and Intelligent Systems (SCIS) and 15th International Symposium on Advanced Intelligent Systems (ISIS), Kitakyushu, 2014, pp. 1213-1218.

[13] R. C. Chepkwony, J. O. Gwendo and P. K. Kemei, "Energy efficient model for deploying wireless body area networks using multi-hop network topology,"International Journal of Wireless \& Mobile Network(IJWMN), vol. 7, no. 5,2015.

[14] A. Maresh Hasan Ali and J. Hanumanthappa, "Quad tree based static multi hop leach energy efficient routing protocol: a novel approach,"International Journal of Computer Network \& Communications (IJCNC), vol.10, no. 1, 2018.

[15] C. Ahn, B. Ahn, S. Kim and J. Choi, "Experimental outage capacity analysis for off-body wireless body area network channel with transmit diversity,'IEEE Transactions on Consumer Electronics, vol. 58 , no. 2,2012, pp. 274-277.

[16] D. Anzai, T. Koya and J. Wang, "Local frequency off-set spatial diversity with pi/4-DQPSK in implant communications," IEEE 25th Annual International Symposium on Personal, Indoor, and Mobile Radio Communication (PIMRC), Washington DC, 2014, pp. 2164-2167. 
International Journal of Computer Networks \& Communications (IJCNC) Vol.10, No.5, September 2018

[17] C. K. Chang, W. R. Lin, Y. Y. Lin and W. J. Liao, "Diversity antenna design for compact devices of IoT uses," 2016 IEEE International Workshop on Electromagnetics: Applications and Student Innovation Competition (iWEM), Nanjing, 2016, pp. 1-3.

[18] M. Marinova et al., "Diversity Performance of Off-Body MB-OFDM UWB-MIMO," in IEEE Transactions on Antennas and Propagation, vol. 63, no. 7, 2015, pp. 3187-3197.

[19] H. T. Nguyen, T. A. Ramstad and I. Balasingham, "Coded pulse position modulation communication system over the human abdominal channel for medical wireless body area networks,"IEEE 23rd International Symposium on Personal, Indoor and Mobile Radio Communications - (PIMRC), Sydney, NSW, 2012, pp. 1992-1996.

[20] N. Promwongsa and T. Sanguankotchakorn, "Packet size optimization for energy-efficient 2-hop in multipath fading for WBAN," 2016 22nd Asia-Pacific Conference on Communications (APCC), Yogyakarta, 2016, pp. 445-450.

[21] N. Promwongsa and T. Sanguankotchakorn, "Delay analysis of ARQ protocol for energy-efficient transmission in WBAN," 2017 International Conference on Information Networking (ICOIN), Da Nang, 2017, pp. 18-23.

[22] J. H. Choi and H. G. Ryu, "A QAPM(Quadrature Amplitude Position Modulation) for low power consumption communication,"International Symposium on Wireless and Pervasive Computing, Hong Kong, 2011, pp. 1-4.

[23] J. H. Choi and H. G. Ryu, "Design and performance analysis of QAPM modulation in multi-path channel,"8th International Conference on Wireless and Optical Communications Networks, Paris, 2011, pp. 1-5.

[24] X. Huang, H. Shan and X. Shen, "On energy efficiency of cooperative communications in wireless body area network,"IEEE Wireless Communications and Networking Conference, Cancun, Quintana Roo, 2011, pp. 1097-1101.

[25] P. M. Paul and A. V. Babu, "Performance evaluation of cooperative communication in WBANs with maximal ratio combining," International Conference on Computing and Network Communications (CoCoNet), Trivandrum, 2015, pp. 627-632.

[26] J. Ding, E. Dutkiewicz, X. Huang and G. Fang, "Energy efficient cooperative relay selection for UWB based body area networks,"IEEE International Conference on Ultra-Wideband (ICUWB), Sydney, NSW, 2013, pp. 97-102.

[27] JJ. Ding, E. Dutkiewicz, X. Huang and G. Fang, "Energy efficient cooperative transmission in singlerelay UWB based body area networks,'IEEE International Conference on Communications (ICC), London, 2015, pp. 1559-1564.

[28] J. G. Proakis, "Digital communications,” 3rd ed. New York: McGraw-Hill, 1995.

[29] M. K. Simon and M. S. Alouini, "Digital communication over fading channel," Wiley:New York, 2000 .

[30] K. Y. Yazdandoost and K. Sayrafian-Pour, "Channel Model for Body Area Network (BAN)," IEEE P802.15-08-0780-09-0006,2009.

\section{APPENDIX}

Appendix A: The evaluation of finite limit integration associated with the Rayleigh distribution A.1 Proof (27)

$$
\frac{1}{\pi} \int_{0}^{\pi / M}\left(1+\frac{c}{\sin ^{2} \theta}\right)^{-1} d \theta=\frac{1}{\pi} \int_{0}^{\pi / M}\left(1-\frac{c}{\sin ^{2} \theta+c}\right) d \theta
$$


International Journal of Computer Networks \& Communications (IJCNC) Vol.10, No.5, September 2018

$$
=\frac{1}{M}-\frac{1}{\pi} \int_{0}^{\pi / M} \frac{c}{\sin ^{2} \theta+c} d \theta
$$

According to [29], we know that

$$
\int \frac{c}{\sin ^{2} \theta+c} d \theta=c \sqrt{\frac{1}{c(1+c)}}\left[\tan ^{-1}\left(\sqrt{\frac{1+c}{c}} \tan \theta\right)\right]
$$

Consequently, it becomes

$$
\frac{1}{\pi} \int_{0}^{\pi / M}\left(1+\frac{c}{\sin ^{2} \theta}\right)^{-1} d \theta=\frac{1}{M}-\frac{c}{\pi} \sqrt{\frac{1}{c(1+c)}}\left[\tan ^{-1}\left(\sqrt{\frac{1+c}{c}} \tan \theta\right)\right]_{0}^{\pi / M}
$$

For $\mathrm{M}=2$, it yields

$$
\frac{1}{\pi} \int_{0}^{\pi / 2}\left(1+\frac{c}{\sin ^{2} \theta}\right)^{-1} d \theta=\frac{1}{2}\left(1-\sqrt{\frac{c}{1+c}}\right)
$$

For $\mathrm{M}=4$, we get

$$
\frac{1}{\pi} \int_{0}^{\pi / 4}\left(1+\frac{c}{\sin ^{2} \theta}\right)^{-1} d \theta=\frac{1}{4}-\frac{1}{\pi} \sqrt{\frac{c}{1+c}}\left[\tan ^{-1}\left(\sqrt{\frac{1+c}{c}}\right)\right]
$$

By applying (A.1.4) and (A.1.5) into (26), (27) can be obtained.

A. 2 Proof of (35)

$$
\frac{1}{\pi} \int_{0}^{\pi / M}\left(1+\frac{c}{\sin ^{2} \theta}\right)^{-m} d \theta=\frac{1}{\pi} \int_{0}^{\pi / M}\left(\frac{\sin ^{2} \theta}{\sin ^{2} \theta+c}\right)^{m} d \theta
$$

According to [29], we know that

$$
\begin{gathered}
\frac{1}{\pi} \int_{0}^{\pi / M}\left(\frac{\sin ^{2} \theta}{\sin ^{2} \theta+c}\right)^{m} d \theta=\frac{1}{M}-\frac{1}{\pi} \sqrt{\frac{c}{1+c}}\left[\left(\frac{\pi}{2}-\tan ^{-1} \alpha\right) \sum_{n=0}^{m-1}\left(\begin{array}{c}
2 n \\
n
\end{array}\right)\left(\frac{1}{4(1+c)}\right)^{n}-\right. \\
\left.\sin \left(\tan ^{-1} \alpha\right) \sum_{n=1}^{m-1} \sum_{i=1}^{n} \frac{T_{i n}}{(1+c)^{n}}\left[\cos \left(\tan ^{-1} \alpha\right)\right]^{2(n-i)+1}\right]
\end{gathered}
$$

where

$$
\alpha=\sqrt{\frac{c}{1+c}} \cot \frac{\pi}{M}
$$

and

$$
T_{\text {in }}=\frac{\left(\begin{array}{c}
2 n \\
n
\end{array}\right)}{\left(\begin{array}{c}
2(n-i) \\
n-i
\end{array}\right) 4^{i}[2(n-i)+1]}
$$

For $\mathrm{M}=2$, we obtain

$$
\begin{gathered}
\frac{1}{\pi} \int_{0}^{\pi / 2}\left(1+\frac{c}{\sin ^{2} \theta}\right)^{-m} d \theta=\frac{1}{2}\left[1-\mu \sum_{n=0}^{m-1}\left(\begin{array}{c}
2 n \\
n
\end{array}\right)\left(\frac{1-\mu^{2}}{4}\right)^{n}\right] \\
\mu=\sqrt{\frac{c}{1+c}}
\end{gathered}
$$

which is equivalent to the form in [28] as shown below:

$$
\frac{1}{\pi} \int_{0}^{\pi / 2}\left(1+\frac{c}{\sin ^{2} \theta}\right)^{-m} d \theta=\left(\frac{1-\mu}{2}\right)^{m} \sum_{n=0}^{m-1}\left(\begin{array}{c}
m-1+n \\
n
\end{array}\right)\left(\frac{1+\mu}{2}\right)^{n}
$$

For $\mathrm{M}=4$, we get

$$
\begin{array}{r}
\frac{1}{\pi} \int_{0}^{\pi / 4}\left(\frac{\sin ^{2} \theta}{\sin ^{2} \theta+c}\right)^{m} d \theta=\frac{1}{4}-\frac{\mu}{\pi}\left[\left(\frac{\pi}{2}-\tan ^{-1} \mu\right) \sum_{n=0}^{m-1}\left(\begin{array}{c}
2 n \\
n
\end{array}\right)\left(\frac{1}{4(1+c)}\right)^{n}-\right. \\
\left.\sin \left(\tan ^{-1} \mu\right) \sum_{n=1}^{m-1} \sum_{i=1}^{n} \frac{T_{i n}}{(1+c)^{n}}\left[\cos \left(\tan ^{-1} \mu\right)\right]^{2(n-i)+1}\right]
\end{array}
$$


By applying (A.2.7) and (A.2.8) into (34), (35) can be obtained.

Appendix B: The Mathematical Proof of the Average BER of Modulation Techniques on Energy Efficiency

In this Section, we compare the performance of four modulation techniques, namely BPSK, 16QAPM, DBPSK and DQPSK in terms of energy efficiency. Here, three communication schemes including 1-hop, 2-hop and Cooperative communications are considered. The average BER is considered as evaluation metric because it is used in deriving the energy efficiency model.

\section{B.1 LOS On-body channel}

In LOS on-body channel, the BER in AWGN channel is used for evaluation instead since they are close to each other. For high SNR, the BERs of all modulation techniques in AWGN channel can be expressed as follows:

$$
\begin{aligned}
P_{b, B P S K}\left(\gamma_{b}\right) & =Q\left(\sqrt{2 \gamma_{b}}\right) \\
P_{b, 16 Q A P M}\left(\gamma_{b}\right) & \approx \frac{5}{8} Q\left(\sqrt{4 \gamma_{b} / 3}\right) \\
P_{b, D B P S K}\left(\gamma_{b}\right) & \geq Q\left(\sqrt{2 \gamma_{b}}\right) \\
P_{b, D Q P S K}\left(\gamma_{b}\right) & \approx Q\left(\sqrt{4 \gamma_{b}} \sin \frac{\pi}{4 \sqrt{2}}\right)
\end{aligned}
$$

By comparing the arguments of $P_{b, B P S K}, P_{b, 16 Q A P M}, P_{b, D B P S K}$ and $P_{b, D Q P S K}$ inside the square root of the Q-function, it is clear that $P_{b, B P S K}<P_{b, D B P S K}<P_{b, 16 Q A P M}<P_{b, 16 Q A P M}$. Thus, $\eta_{B P S K}>\eta_{D B P S K}>$ $\eta_{16 Q A P M}>\eta_{D Q P S K} \cdot$

B.2 In-body channel.

In in-body channel, mathematical proof can be done simply to compare the performance of modulation techniques due to the existence of the simple form of the average BER. It is wellknown in [29] that

$$
\sqrt{\frac{x}{1+x}} \cong 1-\frac{1}{2 x}, x \rightarrow \infty
$$

Consequently, for high SNR, we can approximate the average BERs of BPSK, 16QAPM, DBPSK and DQPSK as follows:

$$
\begin{aligned}
P_{b, B P S K} & =\frac{1}{4 \bar{\gamma}} \\
P_{b, 16 Q A P M} & \approx \frac{1}{4.3 \bar{\gamma}} \\
P_{b, D B P S K} & \approx \frac{1}{2 \bar{\gamma}} \\
P_{b, D Q P S K} & \approx \frac{1}{2}\left(1-\frac{\sqrt{2} \bar{\gamma}}{\sqrt{4 \bar{\gamma}+2 \bar{\gamma}^{2}}}\right)
\end{aligned}
$$

It is apparent that $P_{b, 16 Q A P M}<P_{b, B P S K}<P_{b, D B P S K}$. Next, we prove that $P_{b, D Q P S K}$ is the lowest and approximately the same as $P_{b, D B P S K}$. By taking the limit $\bar{\gamma} \rightarrow \infty$ of the following ratio and using L'hospital's rule, we get

$$
\lim _{\bar{\gamma} \rightarrow \infty} \frac{P_{b, D B P S K}}{P_{b, D Q P S K}}=\lim _{\bar{\gamma} \rightarrow \infty} \frac{1}{(2 / \bar{\gamma}+1)^{-3 / 2}}=1
$$

It is clear that $P_{b, D B P S K}$ and $P_{b, D Q P S K}$ are approximately the same. Therefore, it can be concluded that $\eta_{16 Q A P M}>\eta_{B P S K}>\eta_{D B P S K} \approx \eta_{D Q P S K}$. 